\title{
Habilidades para la vida como estrategia de promoción de la salud en niños y niñas en riesgo social: programa educativo de enfermería ${ }^{1}$
}

\author{
Institución: Universidad de Costa Rica \\ COMO CITAR
}

Angie Moreira González ${ }^{2}$

Paola Murillo Gamboa ${ }^{3}$

Moreira, A. y Murillo, P. (2016). Habilidades para la vida como estrategia de promoción de la salud en niños y niñas en riesgo social: programa educativo de enfermería. Rev. Enfermería Actual de Costa Rica, 30, 1-17. DOI: http://dx.doi.org/10.15517/revenf.v0i30.22114

\section{RESUMEN}

Introducción: El siguiente artículo es el resultado de la investigación realizada para optar por la licenciatura en Enfermería, en la modalidad de práctica dirigida, el cual consistió en un programa educativo desde Enfermería de habilidades para la vida como estrategia de promoción de la salud en niños y niñas en riesgo social del Hogar Vista del Mar en San José, Costa Rica durante el año 2014.

Metodología: participaron 23 niños y niñas en edades entre 9 a 11 años (cuantos del personal de la institución). Se aplicó una metodología cualitativa. Primeramente, se realizó un diagnóstico en el que participaron tanto los niños (as) del programa así como personal que labora para la institución. A partir de los resultados obtenidos en el diagnóstico, se diseñó el programa educativo, desarrollado mediante la metodología de taller con siete sesiones y una actividad de cierre en las que se empleó la estrategia de rally. La evaluación del programa se realizó al finalizar cada una de las sesiones, además de una evaluación general al terminar todo el programa (actividad de rally).

Resultados: Los resultados fueron analizados a la luz del modelo conceptual de sistema abiertos de la teorizante en enfermería Imogene King, entre los que se destaca la importancia de la implementación y el reforzamiento constante de este tipo de programas que, desde Enfermería, proporcionan herramientas para que los infantes cuenten con un mayor control sobre su cuidado, de modo que implementen medidas para promover su salud de manera integral.

Conclusión: Se evidencia la necesidad de reforzar las habilidades para la vida en las poblaciones en condición de riesgo social.

Palabras clave: Habilidades-para-la-vida, niñez-en-riesgo-social, modelo-conceptual-de-sistemas-abiertos, programa-de-Enfermería

\footnotetext{
${ }^{1}$ Fecha de recepción: 27 de agosto 2015

Fecha de aceptación: 2 de octubre 2015

${ }^{2}$ Enfermera. Trabajo independiente. Costa Rica. Correo electrónico: angiem91@hotmail.com

${ }^{3}$ Enfermera. Trabajo independiente. Costa Rica. Correo electrónico: pamga01@gmail.com
} 


\section{Life skills as a strategy for promoting health in children at social risk: nursing education $\operatorname{program}^{1}$}

Angie Moreira González ${ }^{2}$ Paola Murillo Gamboa ${ }^{3}$

Institution: University of Costa Rica

\section{CITED:}

Moreira, A. y Murillo, P. (2016). Life skills as a strategy for promoting health in children at social risk: nursing education program. Rev. Enfermería Actual de Costa Rica, 30, 1-17. DOI: http://dx.doi.org/10.15517/revenf.v0i30.22114

\section{ABSTRACT}

Introduction. The following article is the result of research conducted to opt for a degree in nursing, which consisted of an educational program from Nursing life skills as a strategy for promoting health in children at social risk Home Vista del Mar in San Jose, Costa Rica in 2014.

Methods. 23 children participated in ages between 9-11 years (how many staff of the institution). A qualitative methodology was applied. First, a diagnosis, which involved both, children and staff working for the institution was performed. From the results of the diagnosis, the education program developed by the methodology workshop with seven sessions and a closing activity in which strategy was employed rally was designed. Program evaluation was performed at the end of each session, along with a general assessment at the end of the entire program (activity rally).

Result. The results were analyzed in the light of the conceptual model of open system theorist in nursing Imogene King, including the importance of implementation and constant reinforcement of these types of programs, from Nursing, provide tools for stands that infants have a greater control over their care, so that implement measures to promote their health holistically.

Conclusion. The need to strengthen life skills in populations at social risk condition is evident.

Keywords: childhood-in-social-risk, Life-Skills, Conceptual-Model-of open systems, Nursing-program.

\footnotetext{
${ }^{1}$ Date of acceptance: August 27, 2015

Date of receipt: October 2, 2015

${ }^{2}$ Nurse. University of Costa Rica. Costa Rica. E mail: angiem91@,hotmail.com

${ }^{3}$ Nurse. University de Costa Rica. Costa Rica. E mail: pamga01@gmail.com
} 


\section{Revista Electrónica Enfermeria Actual en costa Rica}

\section{INTRODUCCIÓN}

Como parte de la cultura costarricense, el concepto de salud se relaciona estrechamente con el de enfermedad; sin embargo, como lo mencionan Guerrero y León (2008), la salud va más allá de ese aspecto puramente físico, puesto que también involucra factores psicológicos, afectivos, sociales, cognitivos y espirituales.

Según la Organización Mundial de la Salud (2008), el concepto de salud va más allá de la presencia o no de una enfermedad, definiéndola como un fenómeno con implicaciones sociales, económicas y políticas, además de un derecho fundamental. Al mismo tiempo, agrega que la salud se ve afectada por las desigualdades, la pobreza, la explotación, la violencia y la injusticia, consideradas las causas más importantes de los problemas de salud que, en gran medida, afectan a la población pobre y marginada.

Lo anterior evidencia cómo los factores biológicos y fisiológicos de cada persona no son los únicos que perjudican de manera directa su salud, sino también los sociales, económicos y políticos, lo cual concuerda con Wilkinson y Marmot (2003) quienes señalan que las personas que tienen un mayor poder adquisitivo viven más años y sufren de menos enfermedades que las personas pobres, idea que confirma que los estilos de vida y las condiciones en las que vive cada persona influyen fuertemente tanto en la salud como en su longevidad.

De igual modo, dichos autores destacan cuán relevantes son los primeros años de vida en la salud en la edad adulta, dado que en la niñez es cuando se inicia el desarrollo cognitivo, se esculpe la personalidad y se adquiere y desarrolla habilidades físicas, motoras, sociales y emocionales que permiten enfrentar distintas situaciones en un determinado entorno, por tanto, un crecimiento deficiente y la falta de apoyo emocional en esta etapa de la vida aumentan el riesgo de una salud desfavorable en lo físico, emocional, cognoscitivo y social en años posteriores, particularidad se convierte en la mayor amenaza para la población infantil, ya que de no contar con condiciones sociales y económicas que permitan un desarrollo saludable, se traza una trayectoria social y educacional deficiente para los infantes que aumenta en riesgo psicosocial, lo cual puede afectar su desarrollo integral en cuanto a la probabilidad de iniciar y perdurar en situaciones que interfieren con este, tales como maltrato, descuido, baja escolaridad, explotación económica, vínculos familiares precarios, necesidades básicas insatisfechas, entre otros (Vázquez de Velasco, 2012) que repercuten en la vida adulta.

Dado lo anterior, se rescata la importancia de proporcionarles herramientas psicosociales y emocionales para enfrentarse a los diferentes problemas que presenta el entorno (Mangrulkar, Vince y Poner, 2001), motivo por el que la Organización Mundial de la Salud(2008), promueve las habilidades para la vida como estrategia con la cual, por medio de la adquisición de herramientas psicosociales, los niños, niñas y adolescentes podrán acceder a estilos de vida saludables, a través del fortalecimiento y desarrollo de competencias psicosociales personales (Bravo, 2005). 
La estrategia mencionada agrupa las siguientes 10 habilidades para la vida, fundamentales para infantes y adolescentes en cualquier contexto sociocultural, estas son: conocimiento de sí, empatía, comunicación, efectivas relaciones interpersonales, toma de decisiones, solución de problemas y conflictos, pensamiento creativo, pensamiento crítico, manejo de sentimientos y emociones y manejo de las tensiones y el estrés.

De acuerdo con Melero (2010), tales habilidades se agrupan en tres dimensiones:

1. Aprender a pensar: busca solucionar conflictos sociales por medio del autoconocimiento.

2. Aprender a sentir: involucra los sentimientos humanos y la manera en la que se expresan.

3. Aprender a relacionarse: reconoce al ser humano como un ser social que necesita estar en constante relación con otros.

El fortalecimiento constante de estas tres dimensiones propicia que infantes y adolescentes puedan enfrentarse a las diferentes situaciones de la vida, independientemente del contexto cultural y social en que se desarrollen.

Desde enfermería, este tema es de gran importancia, debido a que es la profesión encargada de velar por el cuidado de las personas en las distintas etapas de su vida, por medio de las habilidades para la vida desde las que se brinda herramientas para que cada persona logre un óptimo estado de salud, a través de las habilidades personales y psicosociales que promueve dicha estrategia; de igual forma, como bien lo señala Pedraza (2011), el profesional en enfermería desde el área pediátrica posee la actitud científica responsable para ejercer liderazgo en el cuidado de la población pediátrica y está en la capacidad de planificar, ejecutar y evaluar programas de salud, con el objetivo de satisfacer las necesidades de este grupo etario.

Por otra parte, realizar este tipo de trabajo permite aplicar las diferentes teorías de enfermería, para así aumentar el valor de esta profesión y el conocimiento que se genera desde esta (Alligood y Tomey, 2011).

El objetivo de esta investigación es desarrollar un programa educativo de Enfermería de habilidades para la vida como estrategia de promoción de la salud, en niños y niñas en riesgo social en el Hogar Vista del Mar, San José 2014.

\section{MATERIALES Y MÉTODO}

Este trabajo se elaboró siguiendo las fases de la práctica dirigida en el Hogar Vista del Mar, Guadalupe, una unidad del Hospicio de Huérfanos de San José. Se contó con la participación un grupo de 23 niños y niñas entre los 9 a 11 años de edad, institucionalizados en dicho hogar y que cumplían con los siguientes criterios de inclusión:

- $\quad$ Ser niño o niña con edades entre los 9 y 11 años de edad. 


\section{Revista Electrónica Enfermeria Actual en costa Rica}

- $\quad$ Residir permanentemente en el Hogar Vista del Mar, Guadalupe, durante el periodo de agosto del 2013 a setiembre del 2014.

La práctica consta de seis fases:

1. Fase de coordinación: en esta fase se realizó las gestiones necesarias para la implementación del programa educativo en el Hogar Vista del Mar (propuesta de trabajo, metodología por utilizar, calendarios),además de una amplia revisión bibliográfica acerca del tema.

2. Fase diagnóstica: se identificó las necesidades de la población respecto de las habilidades para la vida. Para ello se aplicó una entrevista semiestructurada a los niños y niñas y a algunos de los funcionarios del lugar. La entrevista con cuatro funcionarios se realizo para obtener una mayor información de las necesidades que tiene la población con respecto a las habilidades para la vida; este diagnóstico dio paso al diseño del Programa Educativo de Enfermería. Esta etapa responde al primer objetivo específico de este trabajo. Una vez obtenidos los datos, se prosiguió con su análisis a la luz del modelo conceptual de sistemas abiertos de la teorizante en enfermería Imogene King.

3. Fase de diseño del programa educativo de enfermería de habilidades para la vida: a partir del diagnóstico se diseñó el programa educativo, desarrollado con la modalidad de taller y guiado por la teoría sociocultural de Vigostsky.

4. Fase de Implementación del programa educativo en enfermería: se ejecutó el programa educativo, para lo cual, primeramente, se realizaron ocho talleres: el primero fue introductorio, para crear empatía entre las facilitadoras y los (as) menores de edad, mientras que en los seis siguientes se abordó cada una de las seis habilidades para la vida, seleccionadas a partir de la fase diagnóstica; por último, hubo un octavo encuentro en el que se llevó a cabo un rally con la finalidad de poner a prueba las habilidades para la vida que se fortalecieron por medio de los talleres anteriores.

5. Fase de evaluación del programa educativo de enfermería: esta fase se realizó en dos momentos:

- Durante el proceso: al finalizar cada taller, se entregaba a quienes participaron un instrumento que pretendía evaluar las actividades realizadas.

- Evaluación final: se realizó durante la participación en el rally, en el que se valoró la puesta en práctica de las habilidades fortalecidas.

6. Divulgación de los resultados: se redactó el informe final de la práctica dirigida y la defensa pública de este. 


\section{Revista Electrónica Enfermeria Actual en costa Rica}

\section{Consideraciones éticas}

Se consideró los señalamientos establecidos por el "Reglamento Ético científico de la Universidad de Costa Rica para las investigaciones en las que participan seres humanos", entre las que se incluye

- Respeto a la persona y a la personalidad

- La buena fe que expresan los individuos.

- La justicia

- Proporcionalidad y razonabilidad

- La no maleficencia

- La honestidad

Al tratarse de menores de edad, el consentimiento informado fue leído y firmado por la psicóloga de la institución, quien es la representante legal de los menores. Con los niños y niñas se realizó un asentimiento informado en el que se les explicó en qué consistía la fase diagnóstica y el programa, así como si estaban de acuerdo con participar o no. Además, al inicio de cada sesión, se les recordaba que la participación era voluntaria.

\section{RESULTADOS}

A continuación, los resultados de la fase diagnóstica y, posteriormente, los obtenidos tanto en la implementación del programa como en su evaluación.

\section{Resultados de la fase diagnóstica.}

Los datos para esta fase fueron obtenidos a través de los diferentes instrumentos de diagnóstico, aplicados a infantes y funcionarios de la institución. La información recolectada se distribuyó en torno a las categorías definidas para cada una de las seis habilidades para la vida que se incluyó.

\section{Conocimiento de sí.}

Categorías por analizar:

- Imagen corporal

- $\quad$ Fortalezas y debilidades

- Cuidado

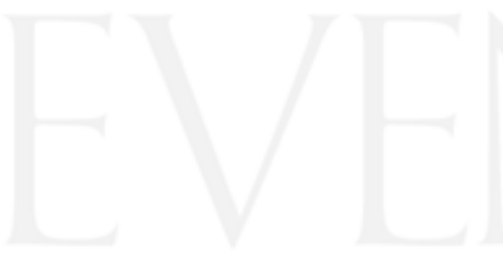

Se indagó con los funcionarios acerca de la capacidad de los infantes para describirse, mas la información brindada no coincide con lo referido por los menores, ya que, ellos y ellas consideran que los niños y niñas son capaces de describirse, lo cual no es así. 


\section{Revista Electrónica Enfermeria actual en costa Rica}

Posteriormente, se cuestionó a los niños y niñas si se sienten a gusto consigo y, aunque la gran mayoría refiere que sí, al preguntarles si les gustaría cambiar algo en ellos, señalan que sí lo harían, refiriéndose a aspectos actitudinales y de crecimiento: P.N²: "no ser malcriado", P.N¹6: "sí, crecer".

Luego, en cuanto a la categoría de fortalezas y debilidades, se observó que las identifican sin dificultad y mencionan, en su mayoría, aspectos actitudinales y académicos: P.ํ2”'estudiar", P.№6: “no me porto bien”.

Por último, en relación con la categoría de cuidado, la población sí realiza acciones en pro de su salud: P.N5: "no como cosas que no son buenas para mí", P.N7: "bañarse y lavarse las manos".

\section{Empatía.}

Categorías por analizar:

- $\quad$ Escucha activa

- Afectividad

- Confidencialidad

Al aplicar la entrevista semiestructurada a los menores, se evidenció que practican la escucha activa con sus pares, ya que la gran mayoría mencionó que escucha a sus amigos cuando les cuentan algún problema. Además, logran identificarse con los sentimientos de otras personas, al mostrarse afectivos, ya que al cuestionarles cómo se sentían ellos cuando un amigo tenía un problema respondieron: P.N¹: "triste por él”, P.N6: "muy mal y me voy para ayudarle".

Asimismo, la confidencialidad es de gran importancia en la empatía: al preguntarles si eran capaces de guardar un secreto, la gran mayoría respondió afirmativo, lo cual coincide parcialmente con lo referido por los informantes claves, ya que en cuanto a confidencialidad, una parte de los funcionarios no consideran que los infantes sean discretos, mientras que la otra parte sí lo considera.

\section{Comunicación efectiva.}

Categorías por analizar:

- $\quad$ Asertividad

- $\quad$ Expresión de sentimientos

- $\quad$ Comprensión del mensaje

Para conocer el manejo de esta habilidad, se formuló cuatro preguntas. La primera pretendía indagar acerca de la forma en la que los menores expresaban sus opiniones cuando no coincidían con las de sus pares, a lo que respondieron: participante $\mathrm{N}^{\circ} 20$ : "le meto un vergaso y me voy", participante $\mathrm{N}^{\circ} 18$ : "no le digo", participante N9: “me enojo con él”. En relación con esta pregunta, pocos refirieron actuar de manera asertiva. 
La segunda y tercera pregunta respecto de dicha habilidad, giró en torno a la identificación y expresión de sentimientos por parte de los menores, por lo que se les cuestionó la manera en la que hacían saber a otras personas cuando estaban tristes o felices y en la que identificaban tales sentimientos en otras personas. A partir de las respuestas, se determinó que, en su mayoría, a los niños les es más fácil expresar sentimientos de tristeza de manera verbal, ejemplo: participante $\mathrm{N}^{\circ} 4$ : "explicándole", participante $\mathrm{N}^{\circ} 10$ : "diciéndoles"; y sentimientos de felicidad de manera no verbal, ejemplo: participante $\mathrm{N}^{\circ} 11$ : "sonriendo", participante $\mathrm{N}^{\circ} 6$ : "porque ando sonriendo".

En cuanto a la manera en que identifican cuando una persona está triste, surgieron diversas respuestas: P. $\mathrm{N}^{\circ} 7$ "porque se le ve en la cara", participante $\mathrm{N}^{\circ} 8$ : "no querer jugar y cuando la llamo para decirle algo y no quiere ir", participante $\mathrm{N}^{\circ} 16$ : "le pregunto"; por ende, se confirma que identifican la expresión de sentimientos por parte de otras personas ya sea de manera verbal o no verbal.

Otra característica de la comunicación efectiva es que el mensaje no solamente es recibido, sino comprendido. Por ello, se indagó las formas por medio del cual los menores determinan que otras personas comprenden lo que les están comentando: según el resultado, la mitad de la población mencionó la escucha activa como el medio por el que reconocen si el mensaje está siendo comprendido, mientras que la otra mitad de la población no identificó algún elemento clave para ello.

Además, al indagar con los funcionarios el manejo y uso de la comunicación efectiva por parte de los menores, indicaron que los infantes -al comunicar determinada situación o comunicar su punto de vista- lo hacen de manera respetuosa y clara, lo cual no coincide con las respuestas obtenidas por parte de los menores, divergencia que podría responder al tipo de relación que mantienen los niños con sus pares y con los funcionarios de la institución. Por último, la mayor parte considera ser capaz de identificar sentimientos y expresarlos a los demás.

\section{Toma de decisiones.}

Categorías por analizar:

- Identificación de problemas

- $\quad$ Búsqueda de soluciones

- $\quad$ Redes de apoyo

Para una adecuada toma de decisiones es necesario identificar el problema, sus causas y las posibles soluciones, motivo por el que al realizar la entrevista semiestructurada a los menores, se les planteó el siguiente enunciado: "Usted está en la montaña, para poder regresar a la casa tiene que cruzar un río, pero el río es muy grande y no existe un puente", a partir del cual debían identificar el problema y posibles soluciones. 
Los datos obtenidos reflejaron que la mayor parte es capaz de identificar el problema, P.N²2: "no hay un puente", P.N9: "no puedo pasar el río"; de igual forma, fueron capaces de proponer soluciones para dicha situación: P.N¹1: "poner un puente", P.N¹3: "con un bote".

También, como parte de esta habilidad, se indagó si los niños recurren a personas cercanas cuando se encuentran en problemas, a lo que la mayor parte afirmó que busca a personas adultas: P.Nº: "a una persona mayor de edad", P.N8: "a una persona de confianza".

Lo anterior coincide parcialmente con la información suministrada por los informantes claves, ya que en relación con la búsqueda de soluciones, los funcionarios(as) refieren que son pocos los menores que identificaron soluciones por ellos mismos.

\section{Pensamiento crítico.}

Categorías por analizar:

$\begin{array}{ll}\text { - } & \text { Autocrítica } \\ \text { - } & \text { Conciencia crítica } \\ & \text { Autonomía }\end{array}$

Dado lo anterior, y respecto de dicha habilidad, se planteó diversos temas a la población, mediante los que se buscaba aplicar la reflexión, el razonamiento y el juicio crítico.

Para investigar acerca de la capacidad de autocrítica, se les cuestionó acerca de la visión que tenían de sí como estudiantes, de lo que se obtuvo respuestas como: P.№6: "como una chiquita muy educada porque la profesora a veces me felicita porque me porto bien", P.N5: "buena, porque me saco 100 en los exámenes". De igual manera, para evaluar la capacidad de conciencia crítica, se les cuestionó acerca de la importancia del reciclaje y de la existencia de situaciones peligrosas para sus vidas. Algunas de las respuestas brindadas fueron: P.N4: "sí, porque es para tener bien el mundo", P.N17: "sí, para no contaminar y no enfermarnos"; P.N8: "andar en la calle sola", P.N²: "cruzar cuando hay carros, ir sola”. Lo anterior evidencia que la gran mayoría es capaz de ser crítica

También se indagó acerca de la autonomía, la cual es indisociable del pensamiento crítico. Para ello, se confrontó a los menores con una situación en la cual debían defender su opinión ante sus amigos(as), con el objetivo de analizar qué acciones llevarían a cabo frente a una realidad similar, algunas de las respuestas fueron: P.N9: "escojo mi opinión", P.N8: "les digo que yo no pienso igual a ellos", P.N²0: "les meto un vergazo, los pateo y me largo". Tales respuestas evidencian que la población procura su autonomía, sin embargo, las estrategias que utilizan para lograrlo incluyen aspectos agresivos.

Respecto de tal habilidad, de manera general, los funcionarios mencionan que los niños son capaces de evaluar sus acciones y sus decisiones; no obstante, evidencian que para la población de 9 a 10 años se constituye 
en un reto mayor. Por otro lado, la mayor parte están de acuerdo en que la población infantil es capaz de exponer y defender sus ideas propias y temas de realidad social.

\section{Manejo del estrés.}

Categorías por analizar:

- $\quad$ Fuentes de estrés

- Identificación de efectos del estrés

- $\quad$ Medidas para reducir el estrés

Fue necesario indagar con la población meta aspectos relacionados con la identificación y el manejo del estrés en medio de su realidad. Para ello, fue necesario, primero, conocer qué entendían los y las menores acerca del concepto de estrés: aproximadamente, la mitad de la población refiere saber acerca del tema, mas no brindó una definición como tal, sino que identifica algunas de las conductas que se manifiestan ante una situación de estrés, por ejemplo: P.N²0: “sí, cuando estoy enojado”, P.N¹3: “sí, cuando se está malcriado".

También, se investigó acerca de las fuentes de estrés dentro del entorno en el que se desenvuelve la población infantil, ante lo que menos de la minoría logró identificarlas, al señalar aspectos como: P.N²1: "que me molesten", P.N²2: “que me molesten, que me digan malas palabras y que me tiren escupa en la cara".

Al cuestionarles sobre las manifestaciones que el estrés causaba en sus vidas, igual que el aspecto anterior, solo la minoría de la población las logró identificar, aludiendo a aspectos como: P.N¹1: "cuando estoy muy enojado", P.N²0: "porque se me repintan las venas".

Por último, se investigó acerca de las estrategias para minimizar el estrés de donde se obtuvo algunas respuestas como: P.N¹2: “me baño para que se me quite”, P.N²1: “contando del 1 al 200".

Al indagar con los funcionarios acerca de esta habilidad, comentan que hay factores causantes de estrés en la cotidianidad de la población. En cuanto a la identificación de los efectos del estrés y al manejo de herramientas por parte de la población meta para el adecuado manejo del estrés, las respuestas fueron diversas, ya que una parte de los informantes claves refieren que los menores no son capaces de reconocer los efectos que causa el estrés en ellos(as) ni cuentan con las herramientas para enfrentar esas situaciones, mientras que la otra parte opina lo contrario.

\section{Resultados del programa.}

Con el propósito de evaluar el impacto del programa, al finalizar cada taller, se realizó una evaluación escrita para verificar la adquisición de nuevo conocimiento por parte de los niños, así como algunos aspectos metodológicos como la satisfacción durante el taller, la metodología utilizada, el tiempo de duración, entre otros. 
Al respecto, es importante destacar que para la implementación de los distintos talleres, se tomó algunas medidas para un mejor aprovechamiento, como las siguientes:

- $\quad$ El grupo se dividió en cuatro subgrupos para un mayor aprovechamiento de las sesiones y para mayor control de la conducta.

- Se procuró que la duración de los talleres no fuera de más de una hora y media (exceptuando el taller \#4).

- Se implementó actividades con características dinámicas, activas y que permitieran la constante participación.

- Además se implementó el uso de reforzamientos positivos, motivó por el que se elaboró un certificado de comportamiento.

A continuación, se describe los resultados según cada habilidad para la vida.

Conocimiento de sí: en este taller la mayoría no identificó aspectos concretos sobre el conocimiento de sí, ya que la mayoría de las respuestas se referían a las actividades realizadas durante el taller y no propiamente a las herramientas brindadas para conocerse .

Empatía: al finalizar este taller, los menores aprendieron satisfactoriamente acerca de este tema. Lo anterior se refleja por medio de la descripción de los contenidos trabajados en los que indicaron haber aprendido a "ponerse en los zapatos de los demás", "ponerse en los zapatos de otra persona" y "no molestar", entre otros.

Comunicación efectiva: al finalizar este taller, los menores indicaron haber adquirido conocimientos sobre la comunicación efectiva; sin embargo, al cuestionarles acerca de los aspectos comprendidos la mayor parte no los describió satisfactoriamente, sino que mencionaban algunos de los juegos efectuados.

Toma de decisiones: en relación con este taller, los menores refirieron haber aprendido nuevos conocimientos acerca de la toma de decisiones, sin embargo, únicamente la mitad de ellos ofreció respuestas satisfactorias respecto de lo aprendido, entre las que se menciona las siguientes: "decidir las decisiones que voy a tomar", "contarle a la encargada", "a pensar", entre otras.

Pensamiento crítico: al cuestionar a los menores acerca del aprendizaje obtenido a través de su participación en este taller, la mayor parte de la población reconoció haber adquirido conocimiento, lo cual fue demostraron al describir de manera satisfactoria algunos aspectos vinculados con el tema, tales como: "pensar de verdad", "pensar sobre el medio ambiente", "verse los errores uno mismo", "defender lo que pensamos", entre otros.

Manejo del estrés: la mayoría de los participantes reconoció haber adquirido nuevos conocimientos, lo cual se evidenció al profundizar acerca de aspectos ligados al tema desarrollado. Al cuestionarles qué habían aprendido 
durante este taller, algunas de las respuestas fueron: "a relajarme", "a relajarme haciendo chistes", "cómo relajarnos", "cómo liberar estrés" entre otras.

Siempre que las facilitadoras lo consideraron necesario, se reforzó los contenidos de aquellos talleres en los que hubo mayor dificultad para adquirir herramientas que permitan poner en práctica las habilidades para la vida. Además, se debe recordar que las habilidades para la vida, más que un constructo teórico, son un conjunto de habilidades y destrezas que se van desarrollando a lo largo de la vida, por ende, su fortalecimiento durante la niñez es de gran importancia para desarrollarlas adecuadamente.

Evaluación del programa (estrategia de Rally): con el objetivo de evaluar los conocimientos adquiridos por los menores a lo largo del programa, se elaboró un rally como estrategia.

Esta actividad se llevó a cabo en la última sesión del programa e incluyó 10 estaciones, cada una con una actividad lúdica y con una pregunta generadora en relación con las habilidades para la vida, de modo que se evaluara el conocimiento adquirido.

En cada una de las estaciones, los menores evidenciaron el manejo de herramientas para emplear las habilidades abordadas, en algunas, con mayor facilidad que en otras. Entre las habilidades que mayor dificultad tuvieron para responder a las preguntas generadoras, se pueden mencionar el conocimiento de sí y el pensamiento crítico. Dado lo anterior, para dichas habilidades, las facilitadoras brindaron una retroalimentación en cada subgrupo.

\section{DISCUSIÓN}

Según Mantilla (2001), las habilidades para la vida son una estrategia de empoderamiento que proporciona a infantes y adolescentes, la capacidad de transformarse como individuos, así como al mundo que los rodea, sin olvidar que tales habilidades permiten reaccionar activamente ante las influencias sociales y ambientales sobre el comportamiento.

Al tratarse de una población en riesgo social, este tema cobra aún mayor relevancia, ya que el fortalecimiento y el desarrollo de las competencias psicosociales de cada persona permitirá a corto, mediano y largo plazo que procuren un estado de salud favorable.

Al observar los resultados de la fase diagnóstica, se observa que, en su mayoría, los infantes poseen estas habilidades; sin embargo, tal como se mencionó, por ser una población en riesgo social es de gran importancia el constante reforzamiento de prácticas para continuar con el desarrollo de habilidades psicosociales, ya que más que un constructo teórico, son prácticas que se van desarrollando y mejorando a lo largo de la vida.

Por otra parte, la aplicación de los instrumentos en la fase diagnóstica evidenció que el conocimiento de sí es una de las habilidades que debe ser reforzada en mayor instancia, debido a que los niños menores presentaron 
gran dificultad para describirse y reconocer la imagen corporal, lo cual se relaciona con los antecedentes de violencia, maltrato, vínculos afectivos precarios y demás que presentan los infantes en riesgo social y que, a su vez, genera problemas emocionales que podrían repercutir en cierta dificultad para reconocerse (González, Ampudia y Guevara, 2012, Fernández y Fernández, 2013).

La relevancia de abordar esta habilidad en este tipo de población radica en que, según Freixa (2003), el autoconocimiento permite a la persona lograr estabilidad emocional y psicológica, particularidad que favorece una autoestima positiva. Además, King (1984) señala que reconocer la imagen corporal, la percepción y el concepto del yo, permite conocerse a sí y sentirse a gusto consigo, aspectos que redundan en el establecimiento y mantenimiento de relaciones interpersonales positivas.

En cuanto a la empatía, los menores evidenciaron ser empáticos con sus pares, sin embargo, al tratarse de una población en riesgo social, la habilidad es aun más trascendental, ya que los niños (as) que son institucionalizados, producto de sus antecedentes, suelen presentar entre sus pares relaciones inseguras, de desconfianza, envidia, rivalidad hostil, otros, por lo que fortalecer esta habilidad permite reforzar relaciones saludables con sus pares.

En cuanto a esta habilidad, los resultados de los instrumentos coinciden con lo referido por Papalia et al. (2010) cuando señalan que, durante la niñez intermedia, los niños toman mayor conciencia sobre sus sentimientos y emociones y logran comprenderlos mejor en otras personas. Lo anterior es fundamental, ya que según Garaiigordobil y García (2006), la empatía permite a los individuos desarrollar pautas de pensamiento y comportamiento acordes con las normas sociales, lo cual, según King (1984), es indispensable para establecer interacciones humanas.

Por otra parte, respecto de la comunicación efectiva, se observó que el proceso de comunicación no es efectivo entre los infantes, debido a que la gran mayoría no logra ser asertiva a lo que se suma que, en ocasiones, hacían referencia a la violencia para expresar su opinión.

En cuanto a la comunicación en los menores, Papalia et al. (2010) refieren que es un proceso que se va desarrollando durante la niñez y que les permite comprender de mejor manera la comunicación verbal y escrita, de modo que esta sea más fácil. Lo anterior es fundamental para la calidad de vida de las personas, en este caso, la población meta, ya que según King (1984) la comunicación es la base de la interacción social y el aprendizaje humano, además de que es el medio para aprender a fortalecer el cuidado y para procurar su bienestar.

Aunado a lo anterior, hay que señalar su componente personal (King, 1984), lo cual se refiere a que cada individuo es diferente y, por ende, se comunica diferente, razón por la que el contexto sociocultural en el que una persona ha crecido y se ha desenvuelto, impacta de manera directa la comunicación. Las condiciones de riesgo social que presenta la población meta de esta investigación apelan a que, desde la enfermería, se aborde dicha 


\section{Revista Electrónica Enfermeria Actual en costa Rica}

habilidad, para que, como bien lo menciona King (1984), la comunicación sea un elemento clave en el establecimiento de relaciones interpersonales sanas que favorezcan el autocuidado y bienestar de los menores.

Ahora bien, en relación con la toma de decisiones, se observó que los infantes son capaces de identificar problemas y sus efectos, lo que coincide con lo referido por Papalia et al (2010), quienes señalan que la población infantil en capaz de correlacionar situaciones con sus causas y efectos, a partir de lo cual toman decisiones más conscientes. Lo anterior convierte esta habilidad en un pilar para toda la vida con el que se enfrenta de manera constructiva las diferentes situaciones que se presentan en la vida cotidiana, ya que, por ejemplo, de no darse una adecuada resolución de conflictos, podría originar afectaciones emocionales, físicas y psicosociales (alcoholismo, consumo de sustancias psicoactivas, otros), aspecto que resalta la importancia de herramientas para la toma reflexiva de decisiones en lugar de las reacciones impulsivas, más aun al tratarse de niños(as) en riesgo social que, por su condición, la toma de decisiones, según Mantilla (2001), puede presentar un mayor reto.

Desde enfermería es esencial reforzar dicha habilidad aun habiendo obtenido resultados positivos en los instrumentos de diagnóstico, ya que como bien lo señala King (1984) diariamente los seres humanos de una u otra forma están tomando decisiones, es decir, están eligiendo alternativas para alcanzar sus objetivos. Asimismo, al ser una población que vive en medio de un entorno vulnerable y con altos niveles de estrés, el no abordarse esta habilidad podría generar problemas sociales como agresividad, delincuencia, aislamiento o alteraciones de tipo emocional, entre otros (Fernández y Fernández, 2013).

En cuanto a la habilidad de pensamiento crítico, se observó gran dificultad por parte de los menores para ser críticos, sin embargo, es lo esperado para esta edad, ya que como bien lo señala Papalia et al (2010), durante esta etapa se presentan avances en el área de pensamiento, juicio crítico y el razonamiento inductivo y deductivo, lo que favorece el desarrollo del pensamiento crítico, el cual se forja a lo largo de la vida, de modo que es necesario reforzar esta habilidad, debido a que incluye aspectos como la criticidad personal y social que propician el respeto por las ideas y opiniones de los demás y la autonomía (Mantilla, 2001). Por lo anterior , se relaciona con la autoridad que posee la persona para tomar sus decisiones de manera consciente y crítica, y además, para dirigir sus acciones en torno a esto, con lo cual, de acuerdo con King (1984), se favorece el logro de objetivos y metas.

Por último, en la habilidad del manejo del estrés se observó que debía ser abordada, ya que debido a las diferentes situaciones en las que se han desarrollado y se desenvuelven los menores es evidente que hay factores que podrían generarles estrés y, como bien lo señala King (1984), el no contar con las herramientas necesarias y adecuadas para hacer frente a este factor, podría tener repercusiones negativas para la salud de los seres humanos, hasta causar , según Fernández y Fernández (2013) un déficit social.

Una vez explicado lo anterior, se recalca la trascendencia del tema de habilidades para la vida, puesto que, desde Enfermería, permite buscar respuestas que satisfagan las necesidades detectadas en la población, como lo 


\section{Revista Electrónica Enfermeria Actual en costa Rica}

fue para este trabajo, proporcionar herramientas a menores, en este caso, en riesgo psicosocial, con el fin de que ejerzan con plenitud su cuidado y así favorecer su salud desde una visión integral.

Además, es necesario un reforzamiento constante de estas habilidades, ya que, si bien es cierto se observan resultados positivos en cuanto a la aplicación del programa y los resultados del rally, también se detectó dificultades en algunas de las habilidades abordadas.

Por último, al realizar este tipo de trabajos, se aplica las teorías de enfermería, lo que incrementa el valor de la profesión, el conocimiento enfermero y justifica su quehacer, mientras combina ciencia, profesión y disciplina.

\section{CONCLUSIONES}

- Al planificar el cuidado de las personas, es necesario contemplar los factores que podrían ser determinantes para su salud, ya que así se enfoca en las necesidades reales de la persona, lo cual aumenta sus oportunidades de éxito. Tomando como base esta idea, es imprescindible trabajar en favor de las habilidades para la vida, principalmente con los niños (as) en condiciones de riesgo social, que además son institucionalizados, ya que representan una población vulnerable, por lo que las habilidades para la vida se convierten en una herramienta esencial para disminuir los posibles efectos negativos de tales condiciones.

- Se determina que la estrategia metodológica de taller, utilizada en este programa, favoreció el aprendizaje de herramientas para fortalecer las habilidades para la vida, puesto que propicia el aprendizaje teórico y ejemplifica cómo hacer uso de estos conceptos en medio de las situaciones diarias. Por otra parte, el uso de la teoría sociocultural de Vigotsky se constituye en una herramienta fundamental al trabajar con población infantil, porque con ella es más fácil conocer la manera en que se aprende por medio de actividades lúdicas, lo cual se maximizó el éxito de este programa.

- $\quad$ Existe la necesidad inherente del fortalecimiento constante de las habilidades para la vida en la población infantil en condiciones de riesgo social.

\section{REFERENCIAS BIBLIOGRÁFICAS}

Alligood, M., Tomey, A. (2011). Modelos y teorías en Enfermería, Barcelona, España: Elseiver.

Báez, C. (2000). La comunicación efectiva. Editor INIEC. Recuperado de

http://books.google.co.cr/books?hl=es\&lr=\&id=dKetaOIuV_sC\&oi=fnd\&pg=PR15\&dq=comunicaci\%C3\%B3n+efectiva\& ots=73yWZ9UjFa\&sig=ym2GTPN7qKLqtI1_r8JQ1BC6qOg\#v=onepage\&q=comunicaci\%C3\%B3n\%20efectiva\&f=false

Bravo, A. (2005). La iniciativa de habilidades para la vida en el ámbito escolar. Revista del instituto de investigaciones educativas, 9 (16), 20 - 29. Recuperado de http://cefire.edu.gva.es/sfp/revistacompartim/arts4/02_com_habilidades_vida.pdf 


\section{Revista Electrónica Enfermeria Actual en costa Rica}

Fernández, M. y Fernández, A. (2013). Problemas de comportamiento y competencias psicosociales en niños y adolescentes institucionalizados. Universitas psychologica, vol $12 \mathrm{~N}^{\circ}$ (3). Recuperado de http://www.redalyc.org/articulo.oa?id=64730275012

Freixa, E. (2003). El conocimiento de sí mismo desde la óptica conductista. Psicothema. 15 (1): 1-5. Recuperado de http://www.psicothema.com/pdf/1015.pdf

Garaigordobil, M. y García, P. (2006). Empatía en niños de 10 a 12 años. Psicothema. 18 (2): 180-186. Recuperado de http://www.unioviedo.net/reunido/index.php/PST/article/view/8490/8354

González, C., Ampudia, A., Guevara, Y. (2012). Programa de intervención para el desarrollo de habilidades sociales en niños institucionalizados. Acta colombiana de Psicología vol (15) $\mathrm{N}^{\circ} 2$. Recuperado de http://portalweb.ucatolica.edu.co/easyWeb2/files/23 9965 v15-n2-art4.pdf

Guerrero, L. y León, A. (2008). Aproximación al concepto de salud: Revisión histórica. Revista Venezolana de Sociología y Antropología. 18 (53). Recuperado de http://www2.scielo.org.ve/scielo.php?script=sci arttext\&pid=S079830692008000300010\&nrm=iso

Instituto Nacional de Evaluación Educativa (s.f.). Mapa reconocimiento y aprecio de sí mismo. Uruguay. Recuperado de http://www.ineed.edu.uy/sites/default/files/NI\%20MP $\% 20$ Reconocimiento $\% 20 \mathrm{y} \% 20$ aprecio $\% 20 \mathrm{de} \% 20 \mathrm{~s} \% \mathrm{C} 3 \% \mathrm{AD} \% 20 \mathrm{mis}$ $\underline{\text { mo.pdf }}$

Jiménez, T., Gutiérrez, A., Hernández, L. y Contreras, C. (2008). Estrés psicosocial: algunos aspectos clínicos y experimentales. Anales de psicología, 24 (2). 353-360. Recuperado de http://www.um.es/analesps/v24/v24_2/19-24_2.pdf

King, I. (1984). Enfermería como profesión. Editorial Limusa. México.

López, M. (2000). Pensamiento crítico [paráfrasis]. Pensamiento crítico y creatividad en el aula. Pág. 87-111. México: Trillas. Recuperado de http://www.uv.mx/personal/cavalerio/files/2011/05/HABILIDADES-CRITICAS-YCREATIVAS1.pdf

Mangrunkar, L., Vince, C., y Posner, M. (2001). Enfoque de habilidades para la vida para un desarrollo saludable en niños y adolescentes. Organización Panamericana de la Salud.

Mantilla, L. (2001). Habilidades para la vida: una propuesta educativa para el desarrollo humano y la prevención de problemas psicosociales. Colombia. Fundación Fe y Alegría. Recuperado de

http://www.feyalegria.org/images/acrobat/72979810510810510097100101115_849.pdf

Melero, J. (2010). Habilidades para la vida: un modelo para educar con sentido. II Seminario de la Red Aragonesa de Escuelas Promotoras de Salud. Zaragoza, España. Recuperado de

http:/www.aragon.es/estaticos/GobiernoAragon/Departamentos/SaludConsumo/Documentos/docs/Profesionales/Salud\%20 publica/Promoci\%C3\%B3n\%20salud\%20escuela/Red\%20Aragonesa\%20Escuelas\%20Promotoras\%20Salud/Jornadas/Un modelo para educar con sentido.pdf 
Mestre, V., Frías M. y Samper, P. (2004). La medida de la empatía: análisis del Interpersonal Reactivity Index. Psicothema. 16 (2): 255-260. Recuperado de http://www.unioviedo.es/reunido/index.php/PST/article/view/8243/8107

Papalia, D., Wendkos, S. y Duskin, R. (2010). Desarrollo Humano. Undécima edición. Bogotá, Colombia. Editorial McGraw Hill.

Pedraza, A. (2011). Aspectos conjuntos profesionales Pediatría/Enfermería. El equipo pediátrico de Atención Primaria: aspectos formativos y asistenciales. Revista Pediátrica de Atención Primaria. (20). Pp. 71-80. España. Recuperado de http://scielo.isciii.es/pdf/pap/v13s20/mesa06.pdf

Vásquez de Velasco, C. (2012). Indicadores para la identificación el riesgo psicosocial de estudiantes. Recuperado de http://www.fad.es/cooperacion/Investigacion IX.pdf

Wilkinson, R., Marmot, M. (2003). Determinantes sociales de la salud: los hechos irrefutables. Recuperado de http://www.bvsde.paho.org/bvsacd/cd90/1008WILdet.pdf 